\title{
CLASSIFICATION OF THE APPROPRIATE BEHAVIORS OF PATIENTS FOR PATIENT SAFETY AGAINST THE CHINESE CULTURAL BACKGROUND, BASED ON GROUNDED THEORY
}

\author{
YAOHUI WANG ${ }^{1,2}$, QUANLONG LIU' ${ }^{1}$, LINA HE ${ }^{2}$, and XINCHUN LI ${ }^{1}$ \\ ${ }^{1}$ China University of Mining and Technology, Xuzhou, China \\ Key Construction Bases of Philosophy and Social Sciences in Jiangsu Universities (Safety Management \\ Research Center), School of Management \\ ${ }^{2}$ Taizhou Hospital of Zhejiang Province, Taizhou, China
}

\begin{abstract}
Objectives: The purpose of this research is to explore the appropriate behaviors and characteristics of Patients for Patient Safety (PFPS) against the Chinese cultural background, especially the types and internal logical relationships of behaviors of PFPS. Material and Methods: In this research, Glazer's methodology principle of the traditional grounded theory was adopted, and the methods of objective sampling, theoretical sampling and snowball sampling were employed. Considering the diversity of the interviewed subjects' gender, age, professional title, qualification and demographic characteristics, representatives of hospital management staff, doctors, nurses, patients and their family members from different provinces and cities across the country were selected for semi-structured in-depth interviews to assess the behaviors of PFPS against the Chinese cultural background. In addition, some PFPS reports were collected from network media to supplement the interview data. All interviews were recorded and collated into Word text documents. Qualitative research data analysis software Nvivo 12 was used to sort out the collected data, and the theme was separated out through the strategy of substantive coding and theoretical coding. Results: In this research, the appropriate behaviors of PFPS were taken as the research content; 6 categories of appropriate behaviors of PFPS were separated out; and a model diagram of PFPS was constructed accordingly. Conclusions: A model diagram of the appropriate behaviors of PFPS against the Chinese cultural background was constructed to provide theoretical guidance for relevant research and practice. Int J Occup Med Environ Health. 2020;33(3):263-72
\end{abstract}

Key words:

behavior, classification, grounded theory, Patient for Patient Safety, medical safety, theoretical guidance

\section{INTRODUCTION}

As a key element of the new medical care, the Patients for Patient Safety (PFPS) initiative has attracted more and more public attention, and this behavior has been advocated as an important way to supervise medical safety.
In March 2006, the World Health Organisation (WHO) issued the "London Declaration on Patients for Patient Safety," emphasizing that patients have the right and responsibility to actively participate in the doctor-patient dialogues and medical activities [1]. Actually, PFPS is one

\footnotetext{
Funding: this research was supported by the Fundamental Research Funds for the Central Universities (grant No. 2019CXNL07, entitled "Construction in safety management research," grant manager: Prof. Xinchun Li), the Project Funded by China Postdoctoral Science Foundation (grant No. 2019M662003, entitled "Effective improvement research of coal mine safety regulation in China from the perspective of risk-based governance," grant manager: Prof. Quanlong Liu).

Received: July 28, 2019. Accepted: December 31, 2019.

Corresponding author: Xinchun Li, China University of Mining and Technology, Key Construction Bases of Philosophy and Social Sciences in Jiangsu Universities (Safety Management Research Center), School of Management, 1 Daxue Road, 221116, Xuzhou, China (e-mail: ezwyh@163.com).
} 
of the main strategies advocated by the patient safety program, the WHO Patient Safety to promote patient safety. The project emphasizes that patients should be recognized for their important role in preventing and protecting patient safety, and encourages patients and their families to actively participate in patient safety activities. It also encourages doctors to listen to and collect patients' and their families' opinions and suggestions on safety through effective methods, so as to assist medical service providers to avoid medical failures as much as possible, and to ensure patient safety [2]. Patients For Patient Safety mainly includes patients' participation in hospital infection control, identity verification, and the prevention of adverse events in medical and nursing safety [3].

In 2017, WHO launched the safe drug use project, aiming to reduce the avoidable drug safety related damage by $50 \%$ in 5 years [4]. Patients can promote drug safety by participating in drug verification, obtaining drug information, and communicating with medical staff in a timely manner [5]. Carman pointed out that patients' participation should be a continuous process of multi-dimensional consultation, participation, cooperation and sharing [6]. In China, the government and researchers have also begun to pay attention to the importance of PFPS and have carried out many studies. Studies from all over the world show that PFPS is generally beneficial to the improvement of patient safety. Some behaviors of patient participation are affirmed, while some others are questioned, by medical personnel [7,8]. Against the Chinese cultural background, what are the appropriate behaviors of PFPS? Amongst the affirmed and encouraged participatory behaviors, can they be classified into categories with their own characteristics? The authors of this study hope that answers to these questions will help patients in China to better participate in patient safety.

This study is aimed to classify the appropriate behaviors of PFPS in China, and to explore their classification characteristics, so as to lay a foundation for further research on PFPS.

\section{MATERIAL AND METHODS}

The purpose of this study is to explore the characteristics and structural types of the appropriate behaviors of PFPS against the Chinese cultural background. When qualitative research was conducted based on grounded theory, the model of the appropriate behaviors of PFPS was constructed mainly by designing non-structured questionnaires to conduct in-depth interviews on typical samples, following which open coding, spindle decoding and selective decoding on the interview data was performed.

The qualitative research performed in this study adopted theoretical sampling, i.e., selecting samples according to the research purpose, which is different from large-scale random sampling used in quantitative research. Based on grounded theory research, relatively few typical cases are selected for an in-depth analysis. Grounded theory requires that the theoretical samples it uses have a certain breadth and depth in data sources [9]. Based on the above principles, this study fully considered the breadth and depth of the data sources in hospital and patient selection, and comprehensively selected some representative interviewees.

In order to participate in patient safety scientifically and effectively, patients need to have certain abilities and conditions. Because this paper is aimed to study the appropriate behaviors of patients participating in patient safety, the interviewees are from the relatively well qualified population, and finally selected pediatrics, obstetrics and orthopedics departments. The patients (or their parents) in these departments are relatively young. The level of knowledge and culture is relatively high. Except for a few severe patients, most people are likely to participate. The determination of the sample size is subject to the principle of theoretical saturation, i.e., samples are extracted until the newly extracted samples no longer provide new important information. In January-March 2019, this study involved 
Table 1. Statistical data of the interviewees - Chinese hospital representatives from different provinces and cities across the country taking part in semi-structured in-depth interviews in the research on the behaviors and characteristics of Patients for Patient Safety against the Chinese cultural background, held in January-March 2019 (Taizhou, Xuzhou, Hangzhou, Nanjing, China)

\begin{tabular}{|c|c|c|}
\hline \multirow[t]{2}{*}{ Variable } & \multicolumn{2}{|c|}{$\begin{array}{l}\text { Participants } \\
(\mathrm{N}=48)\end{array}$} \\
\hline & $\mathrm{n}$ & $\%$ \\
\hline \multicolumn{3}{|l|}{ Gender } \\
\hline male & 26 & 54.2 \\
\hline female & 22 & 45.8 \\
\hline \multicolumn{3}{|l|}{ Age } \\
\hline$<25$ years & 6 & 12.5 \\
\hline $26-35$ years & 26 & 54.2 \\
\hline $36-45$ years & 12 & 25 \\
\hline$>46$ years & 4 & 8.3 \\
\hline \multicolumn{3}{|l|}{ Educational background } \\
\hline doctor & 4 & 8.3 \\
\hline master & 12 & 25 \\
\hline undergraduate & 22 & 45.8 \\
\hline other & 10 & 20.9 \\
\hline \multicolumn{3}{|l|}{ Hospital grade } \\
\hline tertiary & 24 & 50 \\
\hline second & 10 & 20.8 \\
\hline first & 14 & 29.2 \\
\hline \multicolumn{3}{|l|}{ Job } \\
\hline patients and representatives of their family & 22 & 45.8 \\
\hline hospital management personnel & 6 & 12.5 \\
\hline nurses & 4 & 8.3 \\
\hline doctors & 16 & 33.3 \\
\hline
\end{tabular}

conducting in-depth interviews with 48 selected interviewees, and the statistical data of the interviewees are shown in Table 1.

Before the interview, the interviewed subjects and the interview outlines were informed, so as to establish a preliminary connection between the researchers and the interviewees through preliminary communication, and to eliminate some communication obstacles. At the same time, through the preliminary preparation work of the interview, the interviewees could be prompted to summarize and reflect on their own work, and to make certain preparations for the formal interviews.

During the formal interviews, researchers explained the connotation of the appropriate behaviors of PFPS, and then conducted in-depth interviews on the subject. The main interview questions were:

- What do you know about the appropriate behaviors of PFPS against the Chinese cultural background?

- Do you know any patients who have participated in patient safety activities? 
- What do you think are the appropriate behaviors for patients to participate in patient safety activities, and which types can be classified into?

- What do you think are the inherent logical relationships among these appropriate behaviors?

During the interview, the authors also asked the interviewees for explanations based on their responses and some key points.

The in-depth interviews in this study adopted face-to-face approaches, so as to gain the interviewees' trust through face-to-face communication, thus helping the interviewees to express their thoughts without reservation. In order to ensure the quality of the interviews, the authors had no less than $60 \mathrm{~min}$ for each interview and recorded the whole process. After each interview, the interviews were adjusted and optimized in time.

\section{RESULTS}

\section{Open coding}

According to the relevant theoretical requirements of the open coding of grounded theory, combined with the materials collected from the interviews, the authors conducted open coding for the behaviors of PFPS. In order to avoid a subjective bias and established academic conclusions, they also organized theoretical and practical discussions attended by experts. Finally, 27 categories were extracted from the initial concepts, and some examples are shown in Table 2.

Table 2. Concepts and categories formed by open coding for the collected data in the research on the appropriate behaviors and characteristics of Patients For Patient Safety against the Chinese cultural background

\begin{tabular}{|c|c|c|}
\hline Category & Concepts & $\begin{array}{c}\text { Answers } \\
{[\mathrm{n}]}\end{array}$ \\
\hline Confirming treatment plans & $\begin{array}{l}\text { Patients participate in the discussion, on the basis of diagnosis and treatment } \\
\text { combined with individual actual situations, and jointly determine the treatment plan. }\end{array}$ & 55 \\
\hline Selecting anesthesia methods & $\begin{array}{l}\text { Under the established treatment plans, patients determine the operation and } \\
\text { anesthesia methods. }\end{array}$ & 34 \\
\hline Selecting consumables and drugs & $\begin{array}{l}\text { Within the range of options, patients work with doctors to select consumables and } \\
\text { drugs. }\end{array}$ & 24 \\
\hline Signing consent forms & $\begin{array}{l}\text { In the whole treatment process, patients actively participate in and sign various } \\
\text { consent forms on the basis of full understanding. }\end{array}$ & 62 \\
\hline $\begin{array}{l}\text { Determining other treatment } \\
\text { measures }\end{array}$ & $\begin{array}{l}\text { In the case of illness changes or rescues, patients ought to confirm other treatment } \\
\text { measures when medical personnel need to make decisions. }\end{array}$ & 33 \\
\hline $\begin{array}{l}\text { Assisting in checking the quality } \\
\text { of drugs and medical materials }\end{array}$ & $\begin{array}{l}\text { Patients assist medical personnel in the quality inspection of drugs and medical } \\
\text { materials, and replace them if defects are found. }\end{array}$ & 42 \\
\hline $\begin{array}{l}\text { Assisting in checking the quantity } \\
\text { of drugs and medical materials }\end{array}$ & $\begin{array}{l}\text { Patients assist medical personnel in checking the quantity of drugs and medical } \\
\text { materials, and adjusting any surplus and shortage. }\end{array}$ & 26 \\
\hline Process supervising & $\begin{array}{l}\text { Patients supervise medical personnel who have not followed the rules of medical } \\
\text { treatment processes to ensure that they receive treatment under the standard } \\
\text { process. }\end{array}$ & 28 \\
\hline $\begin{array}{l}\text { Cooperating with } \\
\text { the implementation } \\
\text { of operation rules }\end{array}$ & $\begin{array}{l}\text { Patients take the initiative to receive treatment in accordance with the operation } \\
\text { standards in hospitals, and cooperate with following the corresponding operation } \\
\text { rules. }\end{array}$ & 32 \\
\hline $\begin{array}{l}\text { Reminding medical personnel to } \\
\text { operate according to the rules }\end{array}$ & $\begin{array}{l}\text { Patients supervise medical personnel who have not followed the rules of medical } \\
\text { treatment processes to ensure that they receive treatment under standardized } \\
\text { processes. }\end{array}$ & 34 \\
\hline
\end{tabular}


Table 2. Concepts and categories formed by open coding for the collected data in the research on the appropriate behaviors and characteristics of Patients For Patient Safety against the of Chinese cultural background - cont.

\begin{tabular}{|c|c|c|}
\hline Category & Concepts & $\begin{array}{c}\text { Answers } \\
{[\mathrm{n}]}\end{array}$ \\
\hline Observing hospital systems & $\begin{array}{l}\text { Patients arrange escort personnel as required, do not go out without authorization, } \\
\text { and do all kinds of prevention work as required. }\end{array}$ & 45 \\
\hline Taking medicines as required & Timely, adequate and scientific medication. & 23 \\
\hline $\begin{array}{l}\text { Rehabilitation training } \\
\text { as required }\end{array}$ & $\begin{array}{l}\text { Under the guidance of medical personnel, patients complete rehabilitation training } \\
\text { in strict accordance with the requirements. }\end{array}$ & 55 \\
\hline Timely payment & $\begin{array}{l}\text { Patients pay their fees in time, thus ensuring that their medical treatments are not } \\
\text { affected by any arrears. }\end{array}$ & 23 \\
\hline Timely inquiry & $\begin{array}{l}\text { In the case of medical confusion and problems, patients ask medical personnel } \\
\text { for help in time. }\end{array}$ & 45 \\
\hline $\begin{array}{l}\text { Timely reporting of any adverse } \\
\text { events }\end{array}$ & $\begin{array}{l}\text { Any adverse events that have occurred in the medical process are reported } \\
\text { to the hospital timely. }\end{array}$ & 32 \\
\hline $\begin{array}{l}\text { Truthfully reporting medical } \\
\text { history and other discomforts }\end{array}$ & $\begin{array}{l}\text { Patients do not hide anything from medical staff and truthfully report their medical } \\
\text { history. In the treatment process, patients timely report their discomforts and } \\
\text { changes, so that medical personnel can better and faster take corresponding actions. }\end{array}$ & 25 \\
\hline $\begin{array}{l}\text { Prompt acknowledgement and } \\
\text { praising }\end{array}$ & $\begin{array}{l}\text { Patients give acknowledgement and praising for the positive efforts made by medical } \\
\text { personnel. }\end{array}$ & 33 \\
\hline $\begin{array}{l}\text { Encouraging medical personnel } \\
\text { to innovate }\end{array}$ & $\begin{array}{l}\text { Patients encourage medical personnel to innovate when needed and seek } \\
\text { unconventional treatments when necessary. }\end{array}$ & 42 \\
\hline $\begin{array}{l}\text { Taking the initiative to bear } \\
\text { medical risks }\end{array}$ & $\begin{array}{l}\text { Patients should be willing to take medical risks and support medical personnel } \\
\text { to fully rescue and be treated without worries. }\end{array}$ & 30 \\
\hline
\end{tabular}

\section{Spindle coding}

Based on the results of the open coding procedure, the authors further reviewed each category repeatedly, and combined data with medical practice, extracted 6 main categories, including excessive examination, excessive medication, excessive consumables, excessive hospitalization, excessive surgery and malfeasance, especially centered on excessive surgery, thus forming the spindle-type coding as shown in Table 3. The authors further analyzed the behavior characteristics of these 6 main categories.

\section{Selective coding and model construction}

According to the spindle coding performed, the authors analyzed the appropriate behaviors of PFPS, and found that encouraging and supporting behaviors were the basic rela- tionship of mutual trust and run through the whole process. Among them, treatment compliance behaviors, and inquiring and reporting behaviors represent the unilateral behaviors of patients, directly completed by the patients themselves, while procedure-supervising behaviors and standard-reminding behaviors require patients, as the opposite party, to supervise medical behaviors that are not in accordance with the applicable rules, or in violation of these rules, which require skillful efforts to be put forward in a way acceptable to medical personnel. In fact, participatory decision-making behaviors also run through the whole process and reflect the doctorpatient cooperation stipulated by law, which must be pursued jointly by both parties. To this end, the authors constructed a framework model of the appropriate behaviors of PFPS, as shown in Figure 1. 
Table 3. Main category formed by spindle coding for the collected data in the research on the appropriate behaviors and characteristics of Patients For Patient Safety against the Chinese cultural background

\begin{tabular}{lc}
\hline \multicolumn{1}{c}{ Category } & Constituent ratio \\
\hline $\begin{array}{l}\text { Participatory decision-making behavior } \\
\text { determining the treatment plans }\end{array}$ & 20.2 \\
selecting anesthesia methods & \\
deciding on consumables selection & \\
signing consent forms & 18.5 \\
deciding on other diagnosis and treatment measures & \\
Procedure-supervising behavior & \\
assisting in checking the quality of drugs and consumables & 15.1 \\
assisting in checking the quantity of drugs and consumables & \\
process supervising & 22.7 \\
Standard-reminding behavior & \\
cooperating with the implementation of operation rules & \\
reminding medical personnel to operate according to the rules & \\
Treatment compliance behavior & \\
cooperating with inspection & \\
taking medicines as required & \\
recovering as required & 14.3 \\
making timely payments & \\
Inquiring and reporting behavior & \\
timely inquiry & \\
timely reporting any adverse events & \\
truthfully reporting medical history and other discomforts & \\
Encouraging and supporting behavior & \\
timely acknowledgement and praising & \\
encouraging medical personnel to innovate & \\
taking the initiative to bear medical risks & \\
\hline
\end{tabular}

\section{DISCUSSION}

\section{Participatory decision-making behaviors}

In accordance with the provisions of national laws and regulations, patients take the initiative to participate in, or respond to, the requirements of medical personnel, and sign medical plans or diagnosis and treatment behaviors [10], which mainly include treatment plan decisions, surgery anesthesia related decisions, and selecting drugs and consumables. Under Chinese law, doctors must have alternative treatment options for patients, which means that a given patient must make a selective decision from at least 2 schemes. So, patients must discuss and communicate in advance or in time, and tell the doctor their final choices, and ensure that adjustments are not made arbitrarily. It is worth noting that, due to the uneven quality control system of medical institutions, especially in emergency cases, some medical personnel may bring greater risks in recommending treatment programs. In this case, patients do not follow treatment plans as recommended by doctors, and hence unsafe behaviors can be precisely avoided. For patients who need surgical treatment, they usually have to sign consent forms for the operation and anesthesia. For example, some operations can be either conventional or minimally invasive. Although doctors will propose recommendations according to the patients' situation, their orientation of value is generally guided by technology. 
Some surgeries have a variety of anesthesia options and require patients to participate in the decision-making process. In this case, if either doctors or patients do not make decisions from the perspective of patient safety, the risk will be objectively increased. In addition, expensive materials, drugs and implantable consumables also require patients to participate in the decision-making process in order to determine the choice of products from different countries or different manufacturers. The content of patients' participation in decision-making is more than mentioned above, but it is enough to show that among the appropriate behaviors of PFPS, some decision-making behaviors (making choices or signing consent forms) will directly affect patient safety. This is consistent with the requirement of sharing decision-making responsibilities, based on the information sharing between doctors and patients, and on selecting the best care plan according to the individual situation of the patient [11].

\section{Procedure-supervising behaviors}

With the development of modern medical technologies and the establishment of the processes of diagnosis and treatment, especially the treatment of some common and chronic diseases, programmed and process-based treatment plans are often established. However, there may be some unscientific and unreasonable procedures in the implementation processes by different medical personnel. In this event, the role of PFPS is that patients should stop doctors' diagnosis and treatment behaviors in time, report adverse events, and complain to the related hospital department when necessary. For example, the gold standard for tumor surgery is pathological examination. If the patient is diagnosed as having a malignant tumor after examination, some hospitals will arrange the surgery directly. In this case, the patient needs to initiate procedure-supervising behaviors and accept the surgery only after the examination results are available.

In clinical practice, there commonly exist phenomena that the tumors which have been previously diagnosed as malig-

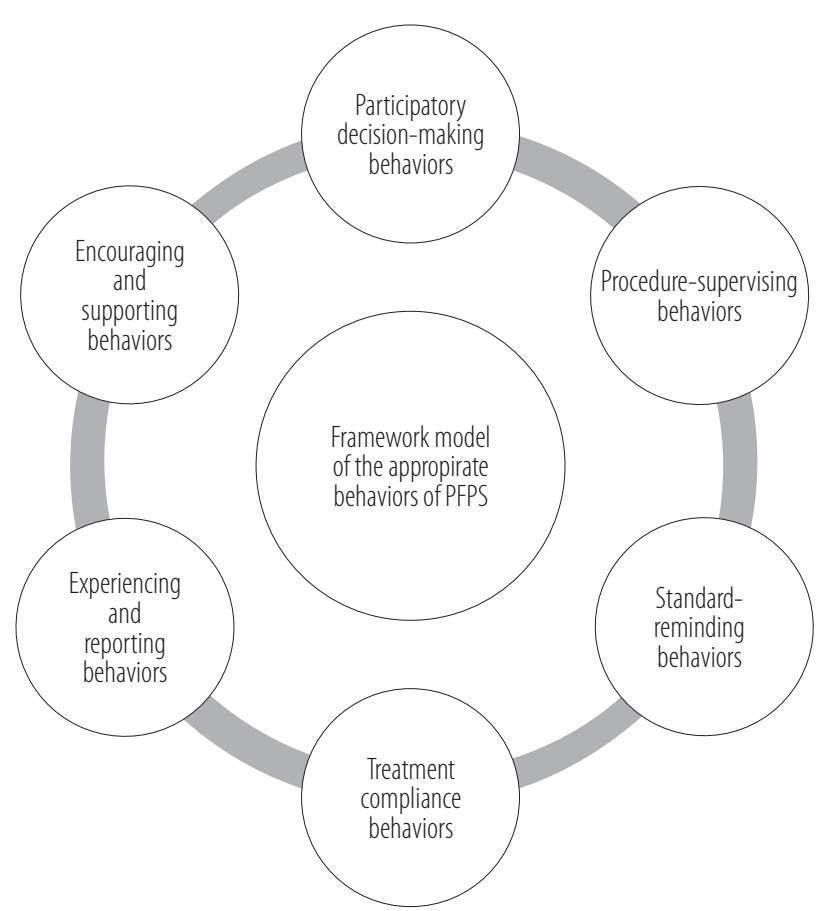

Figure 1. Framework model of the appropriate behaviors of Patients for Patient Safety (PFPS) constructed in the research on the appropriate behaviors and characteristics of PFPS against the Chinese cultural background

nant are confirmed to be benign according to pathological examination, and then treatment plans have to be adjusted. Another example is that for patients who are injected penicillin, some hospitals do not take strict allergy tests for penicillin injections at intervals of more than $36 \mathrm{~h}$. In this case, patients ought to voluntarily ask for allergy tests before receiving penicillin injections. Thanks to these procedural participatory behaviors, the occurrence of small probability events can be avoided. In addition to the patients' supervising and stopping behaviors, the patients themselves must abide by certain requirements during treatment processes, and must not violate the routines of diagnosis and treatment.

\section{Standard-reminding behaviors}

In addition to the corresponding procedures, there is also a large number of industry standards in the treatment process. These standards are often not implemented prop- 
erly in clinical practice, which may have a great impact on patient safety. Namely, patients participate in reminding medical personnel whether they have acted according to the standard. For example, when medical professionals perform diagnostic and treatment procedures on the patient, they can be reminded whether they have washed their hands. Studies have shown that hand washing is the best way to prevent cross infections. Another example is as follows: when taking blood samples, if intern nurses do not operate according to the standard, or if there are any irregular operations, the patient can give the intern necessary reminders, which can effectively reduce the probability of unsafe actions [12].

The most common technical standards that patients can participate in are aseptic operation and isolation. In addition, patients can also participate in the inspection of the validity period of drugs or infusion products, and in the inspection of the quality of infusion products, such as the presence or absence of foreign matters.

\section{Treatment compliance behaviors}

One of the most important aspects of PFPS is to cooperate and comply with the treatment requirements of medical personnel, i.e., treatment compliance behaviors. In the process of hospital treatment, there are many kinds of doctors' orders requiring patients' attention and cooperation, including medication, rehabilitation training and other various matters that need special attention. A strict implementation of these treatment compliance behaviors helps to ensure the safety of treatment and improve the curative effect. Among them, the most common behavior that requires patients to participate is timely, adequate and scientific medication, i.e., the strict drug concentration controlling in some treatment plans, which requires patients to take medicines strictly as required. If they increase or reduce the dosage, or stop taking the medicine without authorization, the treatment effect will be seriously affected, and even side effects will lead to more difficult follow-up treatments.
In addition, patients who are assessed as high-risk falls by medical personnel after surgery should not walk alone as required, and should learn to actively call for help in absence of the accompanying personnel. Many patients will fall down if they do not follow the nurses' requests [12]. There are also patients who leave the hospital without authorization and are unable to deal with emergency conditions due to the decline in their physical function, which gives rise to injuries. Of course, in the clinical treatment process, there are still many behaviors that require patients to actively cooperate or complete, which all require patients to participate well.

\section{Experiencing and reporting behaviors}

It matters whether patients can accurately report their body reactions after the treatment measures in the treatment process. Patients should observe their own body reactions carefully according to the doctors' requirements and individual treatment processes [13], and take the initiative to report good or bad situations. Different patients have different sensitivities to treatment responses, and their timely and accurate reports are helpful for medical personnel to adjust treatment plans in time, and even to discover complications as early as possible. Especially for patients undergoing surgery, if the task of postoperative observation is only left to nurses, complications are often found much later. Some patients are embarrassed to report their physical changes to the medical personnel due to their expressive ability or personality, which will also delay the timely discovery of their illness and corresponding complications. In addition to serious experience, patients should also report timely. Kullberg et al. pointed out that the lack of effective communication between doctors and patients can affect the patients' participation level and safety [14].

\section{Encouraging and supporting behaviors}

Patient safety is the result of the joint efforts made by doctors and patients. Patients participating in patient safety should be trusted and encouraged by medical personnel. Therefore, 
patients should demonstrate encouraging and supporting behaviors, which include making timely payments of fees, being willing to take risks, and supporting the doctors' decisions. Timely payments seem to have little to do with medical activities, but they are regarded by medical personnel as a sign of friendship and support. If patients are unwilling to be responsible for their own medical expenses, they will be perceived by medical personnel as having objections to diagnosis and treatments. In addition, the necessary appreciation activities, including expressing gratitude, presenting pennants and submitting letters of commendation, are also considered as effective encouraging and supporting behaviors, and can attract the attention of medical personnel.

As revealed by this study conducted against the Chinese cultural background, the appropriate behaviors of PFPS can be classified into 6 categories. Encouraging and supporting behaviors are the most basic relationship of mutual trust and run through the whole process. Participatory decision-making behaviors actually run through the whole process and reflect the doctor-patient cooperation stipulated by law, which must be pursued jointly by both parties. In addition, there exist 2 groups of participatory behaviors, i.e., treatment compliance behaviors, and inquiring and reporting behaviors, which are active unilateral behaviors of patients and should be directly manifested by the patients themselves, while procedure-supervising behaviors and standard-reminding behaviors mean that patients, as the opposite party, should supervise the medical personnel's non-standard operation and medical behaviors that violate normal procedures.

In order to improve their own safety, patients need scientific participation in these 6 types of behaviors. For example, in the inpatient treatment of pediatric patients, parents should actively communicate with medical staff to give encouragement and support, and cooperate in the clinical decision-making procedures as required by medical treatment, and should not delay any decisionmaking which could affect the treatment. Then, patients should do a good job in the treatment compliance and experience reporting behavior. In the process of interaction with the medical staff, one should learn to skillfully supervise the procedures and remind warmly, and improve patient safety through participation behaviors.

\section{CONCLUSIONS}

In this research, the appropriate behaviors of PFPS were taken as the research content; 6 categories of appropriate behaviors of PFPS were separated out; and a model diagram of PFPS was constructed accordingly, which will provide theoretical guidance for relevant research and practice.

\section{ACKNOWLEDGMENTS}

The authors would like to express their gratitude to Wanguan Qiao (from China University of Mining and Technology) and Linhong Zhu (from Taizhou Hospital of Zhejiang Province) for their participation and support in grounded interviews.

\section{REFERENCES}

1. Hughes CM, Lapane KL. Nurses' and nursing assistants' perceptions of patient safety culture in nursing homes. Int $\mathrm{J}$ Qual Health Care. 2006;18(4):281-6, https://doi.org/10.1093/ intqhe/mzl020.

2. Doherty C, Stavropoulou C. Patients' willingness and ability to participate actively in the reduction of clinical errors: a systematic literature review. Soc Sci Med. 2012;75(2):257-63, https://doi.org/10.1016/j.socscimed.2012.02.056.

3. King MT, Winters ZE, Olivotto IA, Spillane AJ, Chua BH, Saunders C, et al. Patient-reported outcomes in ductal carcinoma in situ: A systematic review. Eur J Cancer. 2017;71:95108, https://doi.org/10.1016/j.ejca.2016.09.035.

4. Sheikh A, Rudan I, Cresswell K, Dhingra-Kumar N, Tan ML, Häkkinen ML, et al. Agreeing on global research priorities for medication safety: an international prioritisation exercise. J Glob Health. 2019;9(1):010422, https://doi.org/10.7189/jogh. 09.010422 . 
5. Kim JM, Suarez-Cuervo C, Berger Z, Lee J, Gayleard J, Rosenberg C, et al. Evaluation of patient and family engagement strategies to improve medication safety. Patient. 2018;11(2):193-2, https://doi.org/10.1007/s40271-017-0270-8.

6. Carman KL, Dardess P, Maurer M, Sofaer S, Adams K, Bechtel C, et al. Patient and family engagement: a framework for understanding the elements and developing interventions and policies. Health Aff (Millwood). 2013;32(2):223-31, https://doi.org/10.1377/hlthaff.2012.1133.

7. Corina I, Abram M, Halperin D. The Patient's Role in Patient. Safety Obstet Gynecol Clin N Am. 2019;46:215-25, https://doi.org/10.1016/j.ogc.2019.01.004.

8. Cho SM, Choi J. Patient Safety Culture Associated With Patient Safety Competencies Among Registered Nurses. J Nurs Scholarsh. 2018;50(5):549-57, https://doi.org/10.1111/jnu.12413.

9. Handberg C, Thorne S, Midtgaard J, Nielsen CV, Lomborg K. Revisiting symbolic interactionism as a theoretical framework beyond the grounded theory tradition. Qual Health Res. 2015;25(8):1023-32, https://doi.org/10.1177/1049732314554231.
10. Longtin Y, Sax H, Leape LL, Sheridan SE, Donaldson L, Pittet D. Patient participation: current knowledge and applicability to patient safety. Mayo Clin Proc. 2010;85(1):53-62, https://doi.org/10.4065/mcp.2009.0248.

11. Klifto K, Klifto C, Slover J. Current concepts of shared decision making in orthopedic surgery. Curr Rev Musculoskelet Med. 2017;10(2):253-7, https://doi.org/10.1007/s12178-0179409-4.

12. Dunn M. At the moral margins of the doctor-patient relationship. J Med Ethics. 2019;45(3):149-50, https://doi.org/ 10.1136/medethics-2019-105407.

13. Johnstone MJ, Kanitsaki O. Engaging patients as safety partners: Some considerations for ensuring a culturally and linguistically appropriate approach. Health Policy. 2009;90:1-7, https://doi.org/10.1016/j.healthpol.2008.08.007.

14. Kullberg A, Sharp L, Johansson H, Bergenmar M. Information exchange in oncological inpatient care-patient satisfaction, participation, and safety. Eur J Oncol Nurs. 2015;19(2):142-7, https://doi.org/10.1016/j.ejon.2014.10.005.

This work is available in Open Access model and licensed under a Creative Commons Attribution-NonCommercial 3.0 Poland License - http://creativecommons.org/ licenses/by-nc/3.0/pl/deed.en. 